\title{
Marriages of mathematics and physics: A challenge for biology
}

\author{
Arezoo Islami a , Giuseppe Longo ${ }^{\text {b, } *, 1}$ \\ a Stanford University, Department of Philosophy, USA \\ b Centre Cavaillès, CNRS - Ecole Normale Supérieure, Paris and Department of Integrative Physiology and Pathobiology, Tufts University School of Medicine, \\ Boston, USA
}

\section{A R T I C L E I N F O}

\section{Article history:}

Received 12 July 2017

Received in revised form

4 September 2017

Accepted 4 September 2017

Available online $\mathrm{xxx}$

\section{Keywords:}

Geometric vs algebraic constructions Ontological and historical differences Synthesis and applications

Eastern and western traditions

Measurement

Space

Biological evolution and mathematics

\begin{abstract}
A B S T R A C T
The human attempts to access, measure and organize physical phenomena have led to a manifold construction of mathematical and physical spaces. We will survey the evolution of geometries from Euclid to the Algebraic Geometry of the 20th century. The role of Persian/Arabic Algebra in this transition and its Western symbolic development is emphasized. In this relation, we will also discuss changes in the ontological attitudes toward mathematics and its applications. Historically, the encounter of geometric and algebraic perspectives enriched the mathematical practices and their foundations. Yet, the collapse of Euclidean certitudes, of over 2300 years, and the crisis in the mathematical analysis of the 19th century, led to the exclusion of "geometric judgments" from the foundations of Mathematics. After the success and the limits of the logico-formal analysis, it is necessary to broaden our foundational tools and re-examine the interactions with natural sciences. In particular, the way the geometric and algebraic approaches organize knowledge is analyzed as a cross-disciplinary and cross-cultural issue and will be examined in Mathematical Physics and Biology. We finally discuss how the current notions of mathematical (phase) "space" should be revisited for the purposes of life sciences.
\end{abstract}

() 2017 Published by Elsevier Ltd.

\section{Contents}

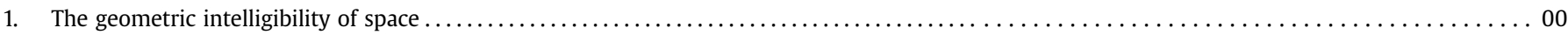

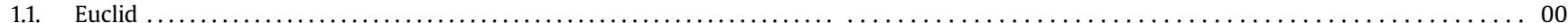

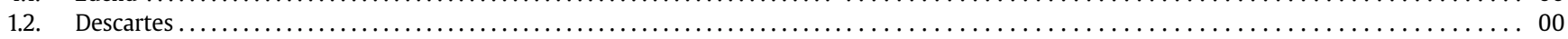

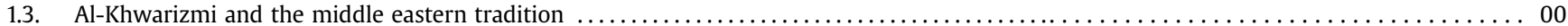

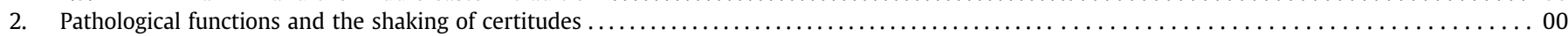

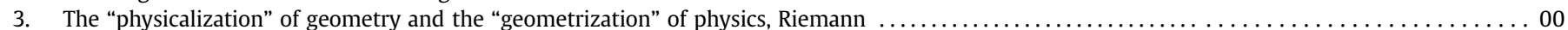

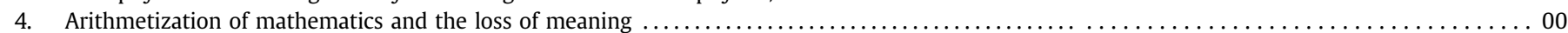

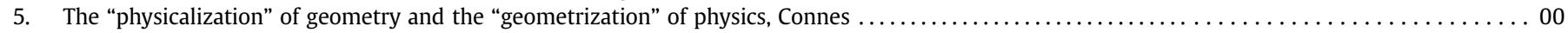

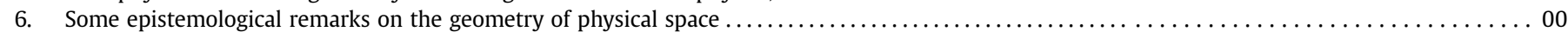

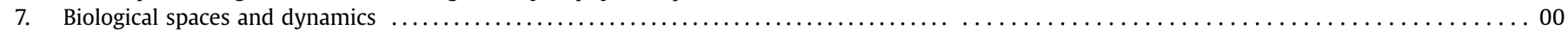

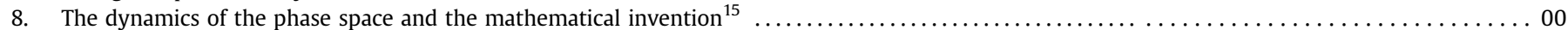

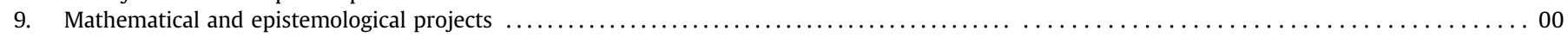

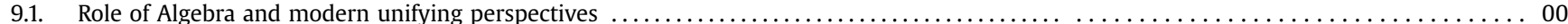

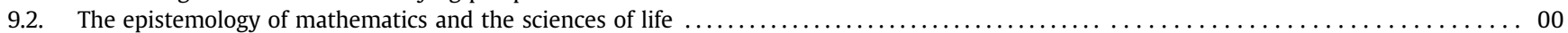

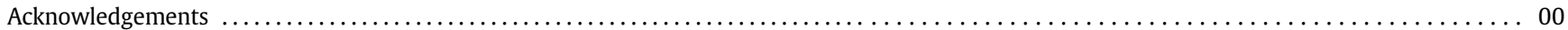

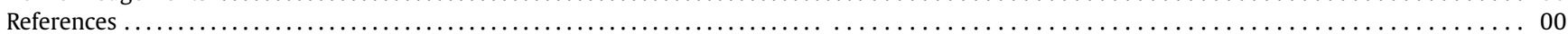

\footnotetext{
* Corresponding author.

E-mail addresses: arezooi@stanford.edu (A. Islami), giuseppe.longo@ens.fr (G. Longo).

URL: http://www.di.ens.fr/users/longo

${ }^{1}$ This author's work is part of the project at IEA, Nantes: “Laws of Gods, Men and Nature": https://www.iea-nantes.fr/rtefiles/File/Projets\%20de\%20recherche/projetgiuseppe-longo-2014_en.pdf.
} 


\section{The geometric intelligibility of space}

«The primary evidence should not be interchanged with the evidence of the "axioms"; since the axioms are mostly the result already of an original formation of meaning and they already have this formation itself always behind them» (Husserl, The origin of Geometry, 1933).

Humans have been since long organizing and giving meaning to space. This was done by action, gestures and language. Mathematics, Geometry in particular, provided the most stable conceptual reconstructions of phenomenal space. By phenomenal space we mean the space on which phenomena manifest themselves. It is the constructed interface, first singled out by our senses, between us and natural processes and on which we draw their contours and dynamics mostly by mathematical tools. We will try to find a methodological unity to the highest moments of this construction, when geometric tools unified, and still now unify, the space of senses and physical space as well as different forms of mathematical understanding of space.

To this aim, and by a rather arbitrary choice, we will stress the unity in the questioning posed by the geometries of Euclid, Descartes, Riemann, and Alain Connes ${ }^{2}$ : the issue here is not the 'names' of the mathematicians mentioned, nor their individual contribution which may interest the historian, but the focus is on the evolution of the notion(s) of space to which these theories have contributed. This will allow us to focus on the issue of the mathematical "space" (or, more generally, "phase space" or space of "possible phylogenetic trajectories") which may be required for the analysis of biological dynamics. The claim is that physical space, in the paradigmatic approaches to geometry, and from our point of view, is made intelligible by proposing different answers to similar "questions": How do we access space? How do we measure it? By which operators do we act on it?

From a philosophical point of view, we aim to analyze the merging of different scientific traditions and to explain the current asymmetry between the role of mathematics with respect to physics on one hand and to biology on the other. While a nuanced historical work on individual mathematicians is important, for the purpose of our project, we focus our attention on the changes in the notion of space that have led to the constitution of physical space using mathematics throughout history.

We base our interpretation of this evolution in part on Edmund Husserl's book The Crisis of European Sciences and Transcendental Phenomenology (Husserl, 1933). In this book Husserl analyzes what he calls the crisis of European Sciences as result of a forgetting of their original constitution since Galileo. In the "Origin of Geometry" he works at the « original » constitutive gestures, in a broad historical perspective, without searching for the actual « protogeometer ». In a similar spirit, we interpret some of the key problems of mathematization of biology as rooted in a forgetting of the very possibility and constitution of mathematical physics and its spaces. We use history as a prism that reveals elements of this constitution as a way to understand origins and thus solutions to our current problem.

History of science in our work is regarded as a continual process that has led to the sciences we possess. We understand each individual's work as a part of this continuum, influencing on and

\footnotetext{
21982 Field Medal, A. Connes works since the early '80's at the geometric
} foundations of Quantum Mechanics. being influenced by the process. Just as any evolutionary analysis, a deeper understanding of a particular moment in history might be gained through a reflection on a later moment, on now in particular.

\subsection{Euclid}

The geometry of Euclid is "just" a geometry of figures: nor space, not even plane are "mathematized". Yet, the developments of Euclidean Geometry provided us the tools to organize physical space by rigid figures, drawn and handled by ruler and compass, and their (rigid) movements. In Euclid's books, figures are drawn and proofs are "shown" (first theorem, book I: construct an equilateral triangle on any straight line ... we all know how ${ }^{3}$ ). In fact, all Euclid's five postulates are "constructive clauses": draw a straight line from any point to any point ... produce a circle with any center and a distance ... and so on so forth. Moreover, each of these construction maximizes symmetries, (see Longo, 2011b); this is the "primary evidence that underlies the axioms" and allows further constructions.

In Greek Geometry the role of seeing is crucial. "Theorem" means "vision", "scene", from "theater", also from the Greek word "theoria" which was what "theoros" saw: theoros, the spectator, the agent of the polis overlooking religious ceremonies from distance without active participation. And so, to solve a mathematical problem, then one has to distance oneself and think about the problem from an impartial point of view. Moreover, symmetry, as "equilibrium" and as a technical notion as well, is at the core of Greek culture, in science, arts and religion. Thus, jointly to constructive gestures in space, such as rotations and translations, which are symmetries on the plane, this notion grounds both the axioms and the proof, by enriching the logical deduction - yet another invention of the Greek Agora.

The famous $\mathrm{V}$ postulate of the parallel lines, for instance, expresses a key invariance property: in modern terms, it is equivalent to "closure under homotheties", as transformations enlarging or reducing length in Euclidean space (its group of automorphisms contains the homotheties). Then, a theorem, a property of a figure, such as the value of the sum of the internal angles of a triangle, remains valid by enlarging or reducing at leisure its length, surface, volume ... from Democritus' atoms to stars. And the "local" or "medium sized" space of senses is perfectly unified with physical space, in the very large and in the very small. This property characterizes Euclidean Geometry with regard to the non-Euclidean

\footnotetext{
3 Trace the circles centered on the extremities of the line, says Euclid (by definition gamma, "the extremities of a line are points"). The intersection point of these circles centered on the end points is obtained by the intended continuity of lines, a well-established notion since the lively debate between Parmenides and Heraclitus in the Greek Agora and the later atomistic response by Democritus. Euclid's lines are the result of a continuous gesture, a construction, a continuous movement with a compass in this case: in this sense, we understand that Euclid's proof are "diagrammatic" and "object dependent", as observed in Panza (2012). Thus, the two lines (a line is a "length with no thickness", definition beta), produce, by intersection, a point, which has "no parts" (definition alpha; a "semeion", a sign, in the original terminology of Euclid). In other words, in Euclid, a point is a position, a semeion, on a line, it is given as the result of an intersection of two lines. We even dare to say that this first theorem at once gives also an implicit definition, or clarification, of what continuity for a line may mean: a line with no thickness is continuous if, when it intersects, in good conditions, another line with no thickness, it produces a point. Only the formalist reading of Greek Geometry could claim that this theorem is not soundly proved by Euclid, according to Hilbertian standards, (see Heath, 1908) and one century long commentaries. Proofs instead are a historical formation of mathematical sense, in this case largely grounded on deep Aristotelian insight into the continuum of movement, (Panza, 2015).
} 
ones.

In summary, in Euclidean geometry, constructions may be done by ruler and compass, thus by rotations and translations (which are symmetries of the plane): these "tools" make figures and measurement possible by a ratio to a given length and, thus, they make space mathematically measurable. These elementary gestures organize space, they allow us to measure it, to operate on it. Nothing else is needed. In this process, geometric objects as ideal objects points, line segments, circle - were constructed, as the result of a mythical construction, the "seeing" ("idein") of objects in a Platonic realm, (Vernant, 1983). So the line as "a length with no thickness" (definition beta) has no physical counterpart, it is a focus on pure measuring. A true origin and foundation of western geometry, it is a limit, an imaginary construction. By the absence of thickness, it allowed an invariant measure of surfaces - what is otherwise the surface of a plane figure with borders of some thickness? Greek Geometry is thus grounded on the invention of the very difficult notion of "border" (see Thom's work on cobordism). Through this ideal, limit language, the subjectivity that is otherwise present in our experience of the world is overcome and replaced with an objective, shareable, understanding of figures, surfaces and, thus and later, of space: the ideal, remote theater looked upon by the theoros. This "objective" knowledge, then, is preserved and passed on through gestures (Châtelet, 1993), speech and writing, from one generation to another, without the need to repeat the original formation of these "pure" objects and their sense (Husserl, 1936). The role of measurement by ratios, the abstract notion of "length", ground Greek geometry in a metaphysics of knowledge that still now play a role in the most advanced mathematical constructions, in particular those which organize space and time.

\subsection{Descartes}

Descartes' geometry may be soundly seen as a conservative extension of Euclid's geometry (Panza, 2011). However, in Euclidean geometry we have at most the construction of the third power, where the first power is a line segment, second a square and third a cube. The higher powers had no geometric equivalent, geometry understood as Euclidean geometry. This problem was solved in Cartesian geometry where a new conception and definition of product as well as extended methods of construction were accepted.

Influenced by algebraic methods developed since the 9th century (see below), Descartes "algebraized" Geometry and founded analytic Geometry (or more accurately, he invented a geometrical algebra where one studies curves defined geometrically). In this Geometry, unlike the synthetic Geometry of Euclid, one does not need to go to higher dimensions to represent the product of two magnitudes. For instance, in Euclidean Geometry prior to Descartes the product of two line segments was considered to be a plane figure, and the product of three line segments a solid and so on. But in Cartesian geometry, it becomes possible to understand the product of line segments as another line segment, thanks to the new definition of product. In this way, it was possible to construct geometrically on a two-dimensional coordinate system every polynomial.

While Euclidian geometers used ruler and compass to construct shapes, Descartes' approach allows to understand "lines" also as the result of a point by point construction or drawing: "If then we should take successively an infinite number of different values for the line $y$, we should obtain an infinite number of values for the line $\mathrm{x}$, and therefore an infinity of different points, such as $\mathrm{C}$, by means of which the required curve can be drawn" [Descartes, 1954, p. 34].

Then Euclidean figures, circles, triangles, ... turned out to be but a special case (at most degree two) of Cartesian curves. Yet, in gaining this amazing power, Geometry took a step away from our ordinary, though abstract, experience of dimension and action in space of senses. Moreover, the correspondence of algebraic formula and geometrical shapes, gave Geometry a symbolic representation, which was much easier to manipulate. That is, the organization and action on space was thus enriched by new algebraic tools. In particular, the correspondence between curves and equations was thought to allow for the geometric representation of not only every polynomial but also eventually every function, after the invention of differential and integral calculus. It was this Geometry that later allowed Newton and Leibniz to develop their ideas of derivative and integral, a new tool for making physical trajectories mathematically intelligible.

\subsection{Al-Khwarizmi and the middle eastern tradition}

What partially made it possible for Descartes to move away from Euclidean Geometry to analytic Geometry was Algebra, which was originally developed in Persian/Arabic tradition ${ }^{4}$ beginning with the work of al-Khwarizmi. While an unsystematic collection of problems and algorithmic solutions existed as early as the Rhind Papyrus c. BC 1700 , and was thought to be an extension of arithmetic in Ancient world and Medieval India, it was not until the work of the mathematician al-Khwarizmi in the 9th century that Algebra was invented as a systematic and rigorous branch of mathematics. This Algebra was not symbolic but verbal, where the problems and their step-by-step solutions were expressed using words. ${ }^{5}$ Unlike his predecessors, al-Khwarizimi did not start with specific problems to solve but with an a priori classification of all equations (linear and quadratic). The book begins by six classes of equations and the introduction of his primitive terms.

The classification of equations is a very important methodological aspect that distinguished al-Khwarizmi's work from prior treatises that used algorithmic methods. The consolidation of Islamic empire in the 8th century led to, among others, the construction of an Arabic dictionary, which involved an "a priori" classification of all possible combinations. Thanks to a theologically neutral view of language, which put the "divine" language of Quran and the language of "pagans" on the same footing, lexicography was done by a priori methods, an art and a science at once. Moreover, the development of civil laws in the 8th century according to Quran's dictum led to the establishment of schools of jurisprudence and the need for new and intricate methods of calculation. Yet once again, art (technique) and science went hand in hand. Algebra's practical purpose did not prevent it from being a science and vice versa. ${ }^{6}$

While Euclid's Elements was concerned with geometric objects in a rigorous logical (deductive) system, al-Khwarizmi's book "al-

\footnotetext{
${ }^{4}$ We use Persian/Arabic, Middle Eastern and Islamic science interchangeably. Given the geographical and historical changes, it is difficult to find the right term for this tradition. Some scholars use "science of the Islamic era", some use "Islamic science", some "Arabic science". But all of these are controversial given the drastic changes in the region. We use Persian not as a term referring to language but to culture. The history of Algebra and its relation to other sciences is rich and very intricate. In this work, we only make a few and rather brief remarks o this matter.

5 Historians often call this verbal Algebra "rhetorical Algebra".

6 Science or knowledge "ilm" in the Islamic tradition has essentially different characteristics than its Greek counterpart. "ilm" is to be understood as that "knowledge" which has practical consequences at some stages of its development. Unlike the Greek tradition, knowledge is not limited to the theoretical and "pure": it is rather oriented toward action. Technique or practical knowledge for Islamic tradition does not hold a lower status comparing to the theoretical knowledge. The aim of "ilm", understood in this way is not describing nature from a distance, as it were, but gaining knowledge by acting upon it (Rosenthal, 1970; Rahman et al., 2008).
} 
jabr and al-moquabala" had a more general purpose: solving problems helpful in Geometry and Arithmetic, which are useful in practical matters such as commerce, distribution, inheritance, law, etc. To solve these problems, al-Khwarizmi invented the new mathematical notion of "shay", the Arabic word for "the thing", "the unknown" (later cosa in Italian) which applied to arithmetical and geometric quantities alike (Al-Khwarizmi, 1915). Note that in the 8th century three major schools for jurisprudence ("Hanafi", "Shafi'i", "Maliki") were developed to (re)construct civil laws about will, inheritance and distribution according to the instructions of Quran. These often needed a complicated calculation and as a result some jurists/mathematicians wrote "hisab al-wasaya" designed specifically for this task. The work of al-Khwarizimi not only further developed and put such calculations on strong, apodictic foundations, but also created a completely new area of mathematics, which contributed to bridge the gap between Arithmetic and Geometry. His work marked the beginning of a six-century long prolific research on Algebra, the relationship between Hellenistic tradition of Geometry and Babylonian Arithmetic and their reconstruction through the application of Algebra (Rashed, 1996). In Algebra, in contrast to Geometry, one does not "see" the proof from distance, but "performs" the calculation in order to arrive at the solution. It is only after carrying out such algebraic operations as "jabr" (restoration/elimination of a negative quantity from both sides of an equation) and "moquabala" (addition of a quantity to both sides) that one reaches the result, and replaces "shay" with its reference.

This tradition was further developed after al-Khwarizmi in two major schools, one arithemetized Algebra and the other geometrized the very same Algebra. As a result of the work of Baghdad mathematician Al-Karaji in "arithmetization", the object of Algebra became polynomial (Rashed, 2015). He extended arithmetical operations to unknowns treating them similar to the known magnitudes. The Persian mathematician, astronomer, philosopher and poet Omar Khayyam in the 11th and 12th century, founded the geometric school and invented methods for solving equations (of degree three) that were not solvable by the method of radicals. He classified all types of third degree equations and also added time to the list of quantities that can be treated algebraically. Starting from arithmetical and geometric magnitudes, Algebra eventually included a treatment of all possible quantities. In the words of d'Alembert in 1751, "Algebra is the method of making general calculations with every kind of quantity by representing them by very universal signs". ${ }^{7}$

Following this new trend, later mathematicians provided for each and every problem two equivalent solutions based on the method of algebraists (e.g. al-Karaji) and geometers (e.g. Khayyam). More importantly, succeeding mathematicians expanded the theory of equations to rational and then to irrational numbers (algebraic numbers, specifically). Moreover to solve cubic equations, they appealed to methods other than construction by ruler and compass and gradually used conic sections (e.g. Khayyam). ${ }^{8}$ Breaking from the ancient prohibitions that kept Arithmetic and Geometry apart, in Algebra, rational, irrational and geometric magnitudes were treated in the same way. This new "algebraic object", "shay" was so general that allowed over time the

\footnotetext{
7 The signs, in Islamic era, weren't yet symbols but terms, such as "shay"(the thing), "mal" (square), and "cab"(cube).

8 As Roshdi Rashed pointed out Khayyam's contributions to the theory of algebraic equations are very significant including "two remarkable results that historians have usually attributed to Descartes: a general solution of all equations of third degree by the intersection of two conics; and a geometrical calculation made possible by the choice of a unit length, keeping faithful, in contrast to Descartes, to the homogeneity rule." (Rashed, 1996).
}

application of Algebra to Geometry, Geometry to Algebra, Algebra to Arithmetic, Algebra to trigonometry, to Euclid's theory of numbers and so on. Thus, this new conception not only broadened the scope of mathematics in a completely unprecedented way (e.g. invention of numerical analysis) but also provided the possibility of its application to itself, cross disciplines.

Another significant difference between this area of mathematics and the Geometry of Euclid was the role of "seeing" in the Platonic realm and the ontological commitment to "pure" objects as the only legitimate objects of mathematics. While in Euclid's geometry what mattered was "showing" and "constructing" by tracing ("producing", translates Heath, 1908), in and as a "theorem", in Persian/Arabic tradition the truth was not necessarily to be grounded on sight. In Algebra, one doesn't see the solution but has a "feeling" for the kind of possible manipulations and operations that might get him to the solution (by an algorithm) (Kvasz, 2008). Although al-Khwarizmi paired every algebraic procedure with a geometric demonstration, as its cause "ila", to ground its certainty, the generality of "shay" and its indifference to the object to which it referred gradually opened the way for purely algebraic arguments, with no need for geometric demonstrations. ${ }^{9}$ We see this method especially in the "arithmetized" Algebra of al-Khwarizmi's successors such as al-Karaji. Transcending from the necessary construction in space opened the way for a new symbolic language in which one was able to write a formula, which encompasses the order of operations as well.

In the 17th century Europe, it was the success of this method that facilitated a new form of "physical abstraction" which was of great importance in the scientific revolution. In the modern physical abstraction one may need to remove "notionally" the material substance e.g. air, as Aristotle could already do, but even gravitation and friction, as Galileo did when imagining the modern inertial movement, the asymptotic invention of the first conservation property. The invention of symbols for operations as well as constants and variables in the works of François Viète and René Descartes gave these abstractions a symbolic representation, which revealed at once the generality of these equations and their algorithmic solutions.

As already hinted, Descartes created a symbolic language representing the unknown by $\mathrm{x}, \mathrm{y}, \mathrm{z}$.., distinguishing the variables from the constants, symbolically. This new language further revealed the "universal" character of algebraic methods, showing that all quantities can be treated in the same way; it was only their relationship that mattered (Kvasz, 2008). Descartes took a step further by declaring that these "accidental", "visible" qualities, as length, as area, as temperature etc., are mere appearances, and the reality hidden underneath is mathematical. He wrote in a letter to Mersenne in 1637: "My entire physics is nothing else than mathematics". The project is a full mathematization of physics as "res extensa", the primary attribute of material substance. In this mathematical space (of coordinates) then the metric was defined as the "distance" between every two points.

\section{Pathological functions and the shaking of certitudes}

The expression of curves by equations allows to establish a point by point relation and, thus, provided a new method of constructing curves. This was thought to allow for the geometric representation of not only every polynomial but also, after the invention of differential and integral calculus, of every function. As Leibniz wrote: "Also if a continuous line be traced, which is now straight, now

\footnotetext{
${ }^{9}$ However, al-Khwarizmi treats irrational quantities arithmetically already in his book and shows that this treatment is justified using geometric methods.
} 
circular, and now of any other description, it is possible to find a mental equivalent, a formula or an equation common to all the points of this line by virtue of which formula the changes in the direction of the line must occur. There is no instance of a face whose contour does not form part of a geometric line and which cannot be traced entirely by a certain mathematical motion. But when the formula is very complex, that which conforms to it passes for irregular." (Leibniz, 1686 p.3).

This paradoxical situation, which later led to the development of fractal (iterative) Geometry and the expansion of the theory of functions of a real variable, had important consequences. It created a general suspicion among mathematicians about the reliability of the intuition they had of these notions. In particular, the correspondence between functions (more precisely analytic formulas) and curves (as their geometric representation) was shaken by the developments in the 19th century. Developments in real analysis, such as the work of Fourier on the heat equation and its solution (Fourier series) led to a class of functions that were not representable by curves: a new intuition, as a novel mathematical insight had to be constructed. While Fourier has proved that "almost" every function is representable by an analytic formula (Fourier series), it gradually became clear that the "exceptions" produce a large class of functions: in fact the "smooth" functions are only a very small class of functions of a real variable. These functions, which were called "pathological" functions, were not constructible geometrically at all, thus defying the strong intuition about the relationship between Cartesian curves and functions (for example, Weierstrass function which is continuous everywhere but differentiable nowhere ${ }^{10}$ ).

Therefore, numerous attempts were made to move away from existing intuition to new insights as a result rigorous proofs and conceptual constructions were produced. In that movement, we see the rise of proofs of existence as opposed to algorithms that gave the solution as a combination of analytic functions. Moreover as a result of Lagrange's work in 1776 it was understood that some differential equations have solutions that are "singular", which cannot be found by standard methods. As a result, Cauchy in his lectures at Ecole Polytechnique, 1821, emphasized the need to prove existence and uniqueness of solutions to differential equations before studying their properties. Instead of attempting to find their solution as an analytical formula, he thought of them as a result of an iterative process, "successive approximations" (Kvasz, 2008). The appearance of these functions in the context of Fourier analysis, and the subsequent work, led to more rigorous definitions of function and integral. In the "refined" definitions, a function does not need to be continuous or constructible by the Cartesian method. In this way, the set of Cartesian curves came to constitute only a small, special subset of the set of all functions. So, Bolzano and Dirichlet dared to define constant functions of dense subsets of the real numbers, such as the rational numbers, acquiring a different value elsewhere. There is no way to "see" or "draw" these pathological functions, even less to relate them to physical dynamics. Then Cantor structured the real numbers by a new understanding of limits and convergence that allowed to frame mathematically these functions. A new mathematical intuition was then constructed, far away from the ancient imagination of the Euclidean "drawn ... produce ... a line".

\footnotetext{
10 This seemed very puzzling since the derivative of a function at a point was thought to give the direction of the corresponding curve at that point. If there were no derivative for the function at any point, how was one to draw or even conceive of the curve? Mathematical imagination had to go beyond Euclid's continuous gestures and trajectories.
}

\section{The "physicalization" of geometry and the "geometrization" of physics, Riemann}

The invention of new spaces for thought was stimulated by a new synthesis, in mathematics. After the marriage of Geometry and Algebra, we now briefly revisit the encounter between Geometry and Differential Analysis, the calculus of derivatives and integrals invented by Leibniz and Newton. Then, in section 5, the triangular relation to Algebra again will bring us to the XX century.

Riemann's main aim, in the 1850 s, is to account for the unexplained Newtonian "action at a distance". He tries to understand gravitation (but electromagnetism and heat propagation as well) by the "structure of space" (Riemann, 1854; see also Boi, 1995; Bottazzini and Tazzioli, 1995). This revolutionary approach may be partly found also in Gauss and Lobachevsky, but it reaches with Riemann its highest mathematical unity, in particular, by generalizing the Euclidean tools for measurement (the ruler and compass) to the "rigid body" (the meter). It may be also seen as an extended Cartesian project for the mathematization of physics, by tools both from geometry and differential analysis. But its physical motivations lead also to a physicalization of Geometry that opened the way to Einstein and, then, to H. Weyl's work, a continual back and forth between (Differential) Geometry and Physics.

One of Riemann's concerns is to understand under which general conditions we may soundly measure. This is possible when rigid bodies are preserved, as by moving a rigid "meter" one may compare lengths. And here comes Riemann's general analysis of curved manifolds, which shows that spaces (manifolds) of constant curvature guarantee the invariance of measurement as the result of the rigidity of bodies. Euclidean space is a particular case of these manifolds, indeed a critical one, i.e. when the curvature is constantly 0 (note also that Euclidean curves were only curves of at most degree two in the Cartesian space).

Yet, the other spaces can make sense as well, since they can give an account, by geodesics, of these mysterious actions at distance. Riemann dares to think that «the concept of rigid body and of $a$ light ray, non longer are valid in the infinitely small» ${ }^{11}$ : bodies may be no longer rigid, light may go along varying curves ... The point is, and this is one of his major results, that the metric structure of a (Riemannian) manifold, or its measurement by a meter, may be biunivocally related to the its curvature (the metric tensor and the tensor of curvature are related, in fully general situations). Moreover, it may make sense to analyze a continuous manifold of nonconstant curvature, as «the foundation of metric relations must be found elsewhere, in cohesive forces that act on it». In other words, forces between bodies are related to the (local-metric) structure of space and, thus, to its (local) curvature. And this approach, writes Riemann, «should be allowed if it would lead to a simpler explanation of the phenomena». A "divination", will recall H. Weyl in the '20s, in reference to Relativity Theory: since Einstein's work, we understand the relevance of this extraordinary insight of Riemann's.

Thus, the new geometric organization of spaces is motivated by physics and may provide an understanding of physical phenomena, beginning with the analysis of measurement and distance. Euclid's ruler and compass is generalized for this purpose, since «... in a continuous manifold the metric relations must be introduced on different grounds». That is, the linear element, which grounds the metric relations, does not need to be represented as the square root of a second order differential form (Pythagoras theorem), but more generally as $\mathrm{ds}^{2}=\Sigma \mathrm{g}_{\mathrm{ij}} \mathrm{dx} \mathrm{x}^{\mathrm{i}} \mathrm{dx}$

\footnotetext{
11 Riemann's quotations, in brackets, are from Riemann (1854).
} 
This is how, for Riemann, we access, we measure and operate on space, while understanding physical phenomena by Geometry. Then spaces (manifolds) are proposed, as a "genealogy of mathematical concepts", by making explicit the hypothesis, which ground the mathematical construction into a new phenomenal space: some key hypothesis are, according to Riemann, connectivity, isotropy, continuity ... H. Weyl will add symmetries and their groups as one of the fundamental properties that structure physical phenomena, (Bailly and Longo, 2011).

Of course, by Riemann's distinction between the "local" and the "global" structure of space (the metric structure and the topological one, the latter related to the Cartesian dimension) a key aspect of the unity of Euclid's approach is lost: physical space, the space of microphysics ("the infinitely small") or astrophysical spaces may have properties which escape our medium size experience of senses and the resulting phenomenal space. In Riemann's approach, the relation between local and global is the result of a complex and novel mathematics: the gluing of local maps by differential methods; homotheties, a group of symmetries, do not allow any longer to transfer "bodily sized" experience and knowledge to any scale. ${ }^{12}$ And this is extremely modern: from Relativity Theory and Quantum Physics, we learned that access, measurement and operations, in the very large and the very small, cannot be provided by the naive analysis of senses. So while in Descartes, and even in Euclidean geometry, we have a physical space that is constructed mathematically and "underlies" the space of human action, with Riemann this is taken a step further, in a more significant separation of the space of ordinary, medium sized objects (space of senses) from those of very large and very small, e. g. in astrophysics and microphysics.

Yet, there is a unity in Euclid's and Riemann's approaches, in their questioning, as described here. A synthesis is beautifully given by Poincaré (1913) in a sentence: «faire de la géométrie, c'est étudier les propriétés de nos instruments, c'est à dire du corps rigide». In short, the tools for measurement are at the core of the geometric constructions.

\section{Arithmetization of mathematics and the loss of meaning}

The development of non-Euclidean geometries, where the parallel postulate was replaced by its negations, added to the overall suspicion regarding direct relation between our geometric constructions and the spaces of senses. Jointly to the invention of "pathological functions" (see sect. 2), this was yet another step away from classical "intuition" and Geometry as both producing and rooted in a unique phenomenal space, the complex result of our action and conceptualization in and of space. Thus, the nonEuclidean approaches to Geometry induced a profound crisis of the 2300 years old Euclidean certitudes, which identified geometry with phenomenal and even physical space, as such: measurement and action here, under the control of our senses and intuition as immediate "seeing", could no longer be extended at all scales (the exclusion of homotheties from the founding principles, the axioms, see below).

Frege's deep investigations started the modern "royal way out" from the novel problem of the gap between intuitive and geometrical (Riemannian) space, between the intuition of movement and trajectories and their mathematical treatment. (Mathematical) Logic was explicitly opposed to foundational analysis grounded on phenomenal space. «The wildest visions of delirium ... remain so long as they remain intuitable, subject to the axioms of Geometry » ... absolute certainty can only be recovered with

\footnotetext{
12 Recall the role of Euclid's V axiom in closing his geometry under homotheties.
}

reference to the concept of number and the logical laws that govern it: «... the laws of arithmetic govern all that is numerable. This is the widest domain of all; for to it belongs no only the actual, not only the intuitable, but everything thinkable» (Frege, 1884, p. 20 and ff.). Geometry itself (but Frege cautiously considers only Euclidean Geometry) can be found analytically on the notion of number, as relation between lengths (Frege, 1873, p. 9-10) (see the discussion in Tappenden (1995)).

In a different way, this program was fully developed by the subsequent work of Hilbert. His first and main foundational writing, (Hilbert, 1899), is a very relevant approach to the issue by formal tools. The foundational problem is reduced to the analysis of formal consistency: what only matters, in mathematics, Geometry in particular, is the non-contradictory status of the axioms, with no reference to meaning, in space in particular. By a remarkable technical work, Hilbert gives all possible "relative consistency" proofs in Geometry: insert an axiom, take another away (Euclidean, non-Euclidean, Desarguesian, non-Desarguesian, Archimedean, non-Archimedean ...) ... embed one system into the other. Beyond Beltrami-Klein's work, the relative interpretations of Lobachevsky's and Riemann's spaces in Euclid's are brought to the highest rigor and generality. Then a final masterpiece: set the basis to formally encode, by analytic tools, Euclidean Geometry in a (conservative, in modern terminology) extension of Arithmetic. The Kantian a priori move from the intuition of (the constructive relation, we would say, to) space and time to the finitistic a priori of Arithmetic counting. The following year in 1900, by posing, at the Paris conference, the problem of consistency of Arithmetic, the scientific program of formal foundation is fully given: no reference to meaning and space, nor to the way we access, by measurement to knowledge of it; just prove formally that the axioms of Arithmetic do not finitely entail " $0=1$ ". This is the foundational problem of mathematics, including Geometry, of course, since the latter, by encoding, is a logical subsystem of Arithmetic.

The extraordinary "tour de force" of Hilbert's is much appreciated by many, including Poincaré. In his review of Hilbert's 1899 book, he acknowledges the technical achievement, but he stresses as well the loss of meaning, the trivialization of our understanding of space, the senseless reference to mathematics as codings of axioms into «le piano raisonneur de Stanley Jevons» from which «on verrait sortir toute la Géométrie». Elsewhere Poincaré, will refer to this view of mathematics, which underlies the foundational programs of Peano, Padoa, Hilbert, as «la machine à saucisses de Chicago»: from pigs and axioms produce sausages and theorems (see Bottazzini, 1999).

\section{The "physicalization" of geometry and the "geometrization" of physics, Connes}

A new synthesis of Geometry and Algebra, this time Abstract Algebra, was brought about through Klein's Erlangen program in 1872. Underlying every geometry, Klein argued, is a symmetry group that is fundamental to the constitution of space. Understood in this way, geometry is the study of the properties that remain invariant under a group of transformations. And the relationship between different geometries must be understood as the relationship between their corresponding groups. This program at once leads to a deeper unity of geometry (Euclidean, non-Euclidean or other) and to a more abstract understanding of space, a step away from the need to fix a metric or to visualize a geometric space. This had far reaching consequences for physics by taking groups, thus abstract symmetries, as underlying the construction of suitable spaces and phase spaces, from Relativity Theory to Quantum Mechanics.

A further marriage relating Algebra and Geometry was later 
proposed by passing through topology. Given any topological and, thus, any metric space $X$, one may consider the set of continuous functions, $C(X)$, from $X$ to the complex field, as a suitable algebraic structure (a commutative $C^{*}$-algebra). $C(X)$ is very important, as it includes the space of measures on $\mathrm{X}$.

A classic result of Gelfand allows to go the other way around. Given a commutative $\mathrm{C}^{*}$-Algebra $\mathbf{C}$, it is possible to construct a topological space $X$, such that $C(X)=C$. The points in $X$ will be characterized by the maximal ideals of $\mathbf{C}$ and so on and so forth as for reconstructing the geometric structure of $\mathrm{X}$ on the grounds of the properties of $\mathbf{C}$.

In classical and relativistic physics measurements happen to commute: the result of different measurement operations does not depend on their order. This is not so in Quantum Mechanics. The measurements of position and momentum, or of time and energy, of a particle do not commute. And this is crucial: in Physics momentum and energy are the key observables, position and time are the associated ("conjugated") parameters. In Quantum Physics, even more than elsewhere, measurement by instruments is the only access we have to "physical reality", that is to the pertinent observables and parameters. More precisely, we can construct empirical knowledge in microphysics only by setting up instruments for measurement. In this, there is a complete conceptual continuity with regard to the approaches by Euclid and Riemann as, in all cases, the tools for accessing and organizing space are the result of a theoretical commitment, they set the direction of the work. But the "instruments" of measurement in Quantum Mechanics do not have the relatively simple nature of the ruler and compass, even not in the generality of Riemann's notion of "rigid body" or of his " $\mathrm{ds}^{2}$ ". Measurement is now given by the complex physical and conceptual instruments of microphysics, and the only "relative" grounding of certainty, which founds quantum mechanics, is given by a few measurable phenomena, where the noncommutativity of measurement has a major role (as well as the related indeterminism, which is intrinsic to the "standard" interpretation). Knowledge construction is then "relativized" as it requires the explicit action of the knowing subject, as well as a strong theoretical project justifying the construction of the instruments. And, in Connes' Geometry, the reference to Gelfand's algebraic approach is at the core of the new geometrization of the phenomenal space of microphysics. Yet, the physical issue of the non-commutativity of quantum measurement introduces a radical novelty as for this new geometrization.

As a matter of fact, as for the mathematics derived from noncommuting measurements, Heisenberg first replaced the algebra of classical mechanics, where observable quantities commute, by a new "calculus of matrices", as it was later acknowledge by Born, where observable quantities do not necessarily commute. In short, his Algebra of matrices is obtained from a groupoid, which replaces the classical (commutative) group of measurements. Now, this latter group gives Connes' starting structure to reconstruct space, à la Gelfand. Thus Connes' work, since many years, consists in reinventing Geometry from a non-commutative ( $\mathrm{C}^{*}$-)algebra: beginning with measurable spaces, to topological, metric and differentiable ones (Connes, 1994). The differences are dramatic, as the very notion of point and of trajectory are different from the classic notions - technically, in non-commutative geometry, there are no more maximal ideals (and trajectories are closer to the "paths" derived from Feynman's notion of path integral). At the core of this, one may find the generalization of the Euclidean key notion of "line" and Descartes' "points". This audacious generalization is the remote heritage of the work by Dedekind and Noether and is at the core of the ideas in Algebraic Geometry, (Patras, 2014).
The debate is very lively (and difficult), but many agree that Connes' approach is gradually giving an account of the mysterious nature of some physical phenomena, at the level of microphysics, including non-locality (a particle is not "located in a point", in Quantum Mechanics). A crucial issue is the dependence of the reconstruction of a possible space for microphysics grounded on the order of measurement: but measurement is how we access to physical space.

In conclusion, beyond Riemann's rigid ruler and the "physical forces acting on/in space", but along the same epistemological lines, also Connes' non-commutative Geometry is grounded on physical properties or, more exactly, on the tools we access and measure physical phenomena. Once more, Poincaré may be quoted for his insight. Even though it would be too much to attribute to him a "divination" concerning the possible Geometry of Quantum Physics, yet he observed: «Des êtres qui éprouveraient nos sensations normales dans un ordre anormal, créeraient une géométrie différente de la nôtre» (Poincaré, 1902).

\section{Some epistemological remarks on the geometry of physical space}

It should be clear that we have no other way to constitute scientific knowledge of nature, but by starting from observable, measurable phenomena, even when this observability has nothing to do with our direct experience by senses. Jointly to the forms of access to and construction of phenomenal spaceq, the algebraization of Geometry and its conjunction to Differential Analysis added more tools for our abstract constructions. As we learned from Relativity and Quantum Physics, we may actually need to give up the identity "(abstract) space of senses = physical space", as the unique phenomenal space for scientific knowledge, so beautifully proposed by Euclidean spaces and their closure by homotheties. Knowledge in very large and very small scales is constructed differently: no rigid ruler, no compass of "human size" may uniformly organize the spaces of galaxies and of quantum particles. Their intelligibility and phenomenality cannot be grounded directly in our senses, on our eyes, hands, by our movements and actions, normalized by Euclid's rigid tools, but must be mediated by complex instruments of observation and measurement. These instruments are themselves the result of complex "theoretical commitments", as they are set up on the basis of an existing or proposed theory, or of strong hypothesis, beginning with the decision to observe and measure "this and not that".

In other words, the dramatic change, in Relativistic and Quantum spaces, is related to cognition: the direct experience of senses is no longer sufficient to make physical space intelligible and unify phenomena. Yet, there is a great unity in the method. It is surprising that we still have to digest this apparent cognitive discontinuity: the "ontological" myth (Geometry is "space per se") does not allow to appreciate that the mathematical objectivity is in the construction and not in an ontology. There is no such thing as "absolute space", but there is the objective reconstruction of a space of action, by the cognitive subject, with the contingent tools of active experience: the result are different phenomenal spaces, in search for a mathematical unity (such as the one between Relativistic and Quantum spaces and their physics), yet grounded on a great methodological unity. Objectivity is reached when the cognizing subject is able to make explicit and relativize these constructions: fix first the tools for measurement, then one or more reference systems or "view points' and a pertinent metrics, thus a scientific "perspective". Then the construction becomes objective by relativizing the perspective, that is by analyzing which are the invariants with regard to the 
transformations/changes of perspective (i.e. of reference system and metrics). As long as the subject believes in absolute spaces (Newton), in "absolute laws of thought" (Frege), in "views from nowhere", there is no foundation for knowledge, but an artificially unified frame for illusory certainties.

In contrast to this, we stress that the method, from Euclid to Riemann and Connes, via Descartes and thus the Persian/Arabic tradition, is uniform and sound: access, measure and operate on space, with the appropriate and explicitly given tools, and organize it by one of our most beautiful conceptual constructions, mathematics, Geometry in particular. Since Descartes, Geometry is a complex blend of constructions of and in phenomenal space and of algebraic abstraction, enriched, by Riemann, by differential tools. Connes' approach, via Gelfand's algebras for geometry, is a further step in this direction: from non-commutative algebras of measurement to an adequate reconstruction of differential geometry, (Connes, 1994).

For a further historical reference to the geometric perspective, note that Poincaré's critique of logicism and formalism proposes to supplement the foundational investigations in mathematics «by a genetic analysis», the analysis of a conceptual genesis or construction (Heinzmann, 1998). His understanding of Geometry as a genesis, beginning with the movements of rigid bodies, specifies Riemann's approach to mathematics as a "genealogy of concepts" as well as Helmholtz's reference to "facts" (see Nabonnand, 2001); it is not an empiricist view nor naively rationalist, but a "phenomenological" understanding (cf. below and (Husserl, 1933), (see Nabonnand, 2000) for more references along these lines).

Mathematics is not grounded on arbitrary conventions: these conventions are the most convenient choices («les plus comodes», writes Poincaré) for us, human beings, in this world, with our shared biological being. Poincaré's program, as we understand it, is a preliminary step to ground mathematics in our reference to the regularities of the world that we see, that we may act on, beginning with symmetries, from Euclid to contemporary Mathematical Physics. In short, we draw mathematics on the phenomenal veil on the grounds of our active, cognitive experience. The structures of mathematics are conceptual proposals, meant to make this world intelligible ( $« \mathrm{Si}$ [la nature] offrait trop de résistance, nous chercherions dans notre arsenal une autre forme qui serait pour elle plus acceptable», (Poincaré, 1899)). As pointed out in (Friedman (2001); p. 26), «Poincaré's ... “conventions" [are] free choices of our own needed to bridge the irreducible gulf between our crude and approximate sensory experience and our precise mathematical descriptions of nature».

The role of acting, proposing, and understanding is crucial. The resistance of nature, its friction against our active knowledge construction, is deeply embedded in its specific materiality and in our contingent biological and cognitive being, in the historical formation of sense. The point is, let's admit it, that "any constitution is contingent" and the historical constructions of mathematics do not escape from this. Yet, objectivity is reached by the methodological unity (invariance, one should say) of the constructions and its relativization: the Greek "theoros" must step back from his/her view point, look from a distance, not from "nowhere", but from an epistemological and historical perspective, by a critique of the method that allowed him/her to propose the view point and grasp what is invariant w.r.to changing perspectives - this is their possible methodological unity.

The foundational program we are sketching here is an epistemological one: it is an analysis of "how" we construct knowledge, or of the "knowledge process". In mathematics, spaces, objects and structures are constructed from the explicit assumption of cognitive grounds, beginning with the reference to the tools for measurement, a key step in the construction of objectivity. Asymptotic constructions, such as the Euclid's "line with no thickness", which need language and myths, then other symbolic forms, such as Algebra and the asymptotes of Differential Analysis, bring further, in the communicating community, towards new invariants the objectivity of the construction, well beyond the subjective access to phenomena.

This constitutive analysis has been programmatically disregarded by the logicist and formalist approaches to the foundation of mathematics, in the XX century, as they only focused on (logicoformal) proofs. This was a necessary investigation, but, unfortunately, it excluded the analysis of the constitution of concepts and structures, from Euclid's line and its transformations by symmetries (rotations, translations) to Poincaré's, Weyl's and Grothendieck's approaches, beyond the ones we hinted to in this text (see Zalamea, 2012; Longo, 2015). The logical and formal approaches pretended to found and encode the world on integer numbers, viewed as "an absolute concept", as in the Fregean tradition, or on countable strings of meaningless symbols, following Hilbert. Now, there is no doubt that mathematics is $a b$ stract and symbolic, but the one century long identification of these two deep notions with a "logic of integers" or with "formal" excluded epistemology and then meaning from the foundational analysis. We have to broaden the foundational project to the "constitutive path" of mathematical abstract structures, beginning by their meaningful grounding in (and their organizing) phenomenal space and time. These are proposed at the interface between us and "reality" by an active construction of concepts and structures, a result of both gestures and actions in space and of language, including the language of Algebra.

\section{Biological spaces and dynamics}

Through the mathematical organization of phenomenal space we constructed, along history, the mathematical spaces of physical dynamics. Let's now focus on life processes and on some mathematical challenges posed by them. These processes are first of all a spatio-temporal matter. The dynamics of forms is at core of biology, beginning with the three dimensional structure of DNA and the folding - unfolding of proteins, which massively depend on dimensions and physico-chemical contexts. Proteins are not "alive", but they are the "bricks of life" - yet, in no way, by knowing all physico-chemical details of bricks one understands, say, the architecture of a building.

The analysis of the spatial organization is the first step towards appreciating the complexity of structures whose functionality is entirely lost by any sort of "linear encoding", such as the description on the tape of a Turing Machine, with its key distinction between hardware and software. Turing first, in his 1952 morphogenesis paper, analyzed the non-linear dynamics of an action/reaction/ diffusion system as an at least two dimensional continuous process, that he opposes to his own "discrete state machine", whose deterministic dynamics reminds him of Laplace's predictability, (Turing, 1950). Turing's analysis is grounded on the sensitivity to border conditions of the system on "the onset of instability", which unfolds by "catastrophic instabilities" in continua and by dynamics deformations of a purely material hardware. This "falsifies", says he, the need of instructions to "trigger" a purely bio-physical process (no "new hypothesis"), (see Turing, 1952) where he quotes, as biologists, only Child, D'Arcy Thompson, Waddington by their 
interest in continuous dynamics of biological forms and patterns, far from any genocentric views, thus from the encodings on discrete data types that he had invented.

Moreover, beyond the essential three dimensionality of organisms and their intrinsic materiality, ${ }^{13}$ living entities also require multi-scale analyses. In some cases, living entities may develop fractal structures and the mathematics of fractals is partly multiscale. Starting at one level of "magnitude" one may go to finer and finer insights into phenomena, at different scales. But the law is just one, indefinitely iterated. As we mentioned in sect. 2, the arrival at fractal geometry itself is a result of the appearance of pathological functions in mathematics, which could not be treated with the usual methods of analysis.

There exist very effective descriptions of vascular and respiratory systems as fractals (see Brown and West, 1999; Nonnenmacher et al., 1994; Bailly et al., 1994 for example). Maximizing exchange surfaces and irrigation volumes yields a mathematical law that gives the general pattern. These are peculiar situations where life forms are organized by a "forced" growth phenomenon, an expanding tissue under a physical constraining force, and the analysis may be largely physical, as for the wax in a beehive or the shells of some invertebrates. Yet, tissues and organs are made out of cells that reproduce with variation, the first principle in Darwin's approach, they are not just "soft matter" shaped by forces, like wax or shells. Then biological variation steps in and requires a finer analysis, (see Montévil et al., 2016b). In particular, the "irregularities", due to variability and diversity in biological fractals, both within an organism and between different organisms in a species, may be functional, as they contribute to adaptivity (Montévil et al., 2016a). We thus need to enrich the mathematical analyses that guided our invention of physical spaces, with their dimensional and, possibly, fractal properties. One of the challenges then would be to integrate the categorical/hierachical approach in (Ehresmann and Vanbremeersch, (2007), Simeonov and Ehresmann (2017)) with the appreciation of the "specificity" of the biological object vs the "genericity" of the categorical one, of the role of adaptivity grounded on diversity and variability, of "constraints" in canalizing biological randomness, from bio-resonance to symmetry breaking, as described in Longo and Montévil (2014), Soto and Longo (2016).

As an informal and preliminary attempt toward this aim, let's just examine one of the aspects mentioned and view the living as an alternating hierarchy of at least two organization levels: autonomous biological individuals (cells), functional groups of them (organs), which in turn are integrated in a higher level and unity by their physiological functions (and yield a new living unity). In (Bailly et al., 1994; Longo et al., 2012), it is observed that, in physico-mathematical terms, fractals geometries can be typically found in organs and are related to their function, that is the exchange of energy or matter (or of their gradients), while the interactions of biological units may be abstractly associated to dynamical systems and be embedded in categorical/relational hierarchies, (Simeonov and Ehresmann, 2017). That is, mathematics, by Fractal Theories, may give a good account of the relation between structure and function in organs (lungs, vascular system ...), while, as for living units, this relations are better analyzed, whenever possible, in terms of dynamical and physiological relations and

\footnotetext{
${ }^{13}$ Organisms are made of a specific physical matter: the bases of DNA, the molecular components of membranes have no alternatives, in a space that we strictly understand in three-dimensions. Synthetic biology extracts and re-combines fragments of DNA, or their exact chemical replica, and places them in cellular membranes with their proper physico-chemical and dimensional structure. The dualistic perspectives, software vs. hardware, or soul vs. body, are a fantastic invention for the purposes of computing with machines, or a strong religious
} commitment, respectively, but do not need to be projected on nature. constraints, (see Montévil and Mossio, 2015). But an organ is composed by tissues that are generated by cells, which, as all organisms, reproduce with variation, (Montévil et al., 2016a). Then, the physical forces, e.g. the flow due to the pumping hearth, constrain cellular reproduction. That is, those physical forces are not the "primary motor" of the fractal structure of, say, the vascular system: they canalize and constrain the proliferating and moving cells (an original analysis based of this "reversing" of principles is in Montévil et al. (2016b)). Note also, that variability is at the core of biological diversity that is a fundamental component of the stability of life: diversity in an individual organ, or between the same organ in different individuals, as we said, contributes to the stability by adaptivity of an individual or of a population, (Montévil et al., 2016a; Longo, 2017).

Note, that even within cells, the least living entity, one may find organs: the external membrane and the cytoscheleton, first, but also some sort of internal membranes and "rails", microtubules, that play a key role in organizing cells' metabolism and reproduction. Their formation as well must be analyzed as constrained reproduction with variation: in recent experiments in microgravity, proliferating yeast cells produce very irregular cytoscheleta, (Bizzarri et al., 2014). On Earth, gravitation constrains their formation - and (negatively) selects them when they are not viable in our gravitational field.

This is just the beginning of a view of complexity of organisms where the mathematical tools commonly used are already split into different theoretical frames, according to the "scale" - cell, organ, organism; each may have some descriptive value, but just for its level of investigation. Moreover, these (already schematic) levels, interact vertically and thus yield a novel, essentially multiscale system: when the scale changes, the mathematics we use for its analysis changes as well (typically, from networks analysis of cells to fractal structures of organs) and these nested levels are integrated and regulate each other. The causal analysis needs to be modified with regards to a purely physical dynamics: as we observed, first (cellular) reproduction with variation, then physical (and biological) constraints acting on or canalizing, constraining reproduction. But this requires an ongoing theoretical construction which is the aim of the work hinted in Soto and Longo (2016).

Finally, "enablement", i.e. the analysis of "what makes possible" a given process, should be added to the analysis of causality, (Longo and Montévil, 2014). For example, enabling contexts give the major differences between in vivo and in vitro experiments. In a neuron, say, the artificial fluid of an in vitro experience and its being cut off from three-dimensional connections etc. give lower firing rates, higher resistance, unreliable potentials ... (Jennings and Aamodt, 2000). In these cases, the arbitrariness of the physicomathematical modeling, a further abstraction from the context, is even greater. The "intended" assumptions are very numerous and mostly out of control, as most are implicit: in an organism almost everything is correlated to almost everything - de-correlation analyses, as the ones done in physics, are extremely hard, sometimes impossible. Sooner or later the researcher will acknowledge that there are "hidden variables" not taken into account in the model; often, this is due to interactions with other scales, out of the scope of the given model. Thus, in contrast to Physics, Models in Biology are always poorer than phenomena, or, more precisely, the powerful correlations between the "genericity" of the mathematical and of the physical object (and "specificity" of trajectories) is lost (is actually reversed) in biology, whose objects are specific (historical) and trajectories are "generic" (possible ones), (see Bailly and Longo, 2011; Longo and Montévil, 2014) for a detailed analysis. All of this stresses the need for a proper "biological theorizing" and a critical perspective in modeling, based on biologically sound 
theoretical principles. ${ }^{14}$

\section{The dynamics of the phase space and the mathematical invention $^{15}$}

All these issues (context dependence, multiscale interactions) pose major challenges to mathematics in Biology, as theoretical generality is also our aim. In Physics, this is "more easily" obtained by mathematics' constitutive role in drawing the very "conceptual contours" of physical objects and by the possible or discernible context independence of physical experiences, while both conditions essentially fail in Biology. Thus, the gap between the "local" nature of Models and the required "global" nature of Theories is much greater than in Physics. Moreover, Mathematical Biology seems to provide only models, so far - to be evaluated critically (see the footnote and reference above). As a matter of fact, even modeling, which is so important for iterating experiences, transferring knowledge ... conjecturing Theories, is very hard. Consider "latent potentials" in Evolution. There is, for example, strong paleontological evidence that the double jaw of some reptiles, living 200 millions years ago, originated the inner ear of birds and mammals (Gould, 1982, 1989). How can you model this? Which energy is minimized, if any, or which geodetics, in which mathematical space one may simulate such a contingent evolution? There exist dynamic models of co-evolutive systems, as they are called, but, before discussing the problems they are faced with, let's consider another, related, feature of life.

In Physics, we know how to deal with states close to as well as far from equilibrium; but also critical states are well defined and treated. By definition, the latter are "temporary": a physical system doesn't stay long in a critical state (on the verge of a change of state). Yet, living entities, both biological units and species, permanently live in an "extended critical state", (Bailly and Longo, 2011). Waddington's homeorhesis or Varela's autopoiesis, (Petitot et al., 1999), are early theoretical appreciation of this fact; where homeorhesis means a dynamical reconstruction of an ever changing equilibrium, which is autopoietic when it is internally reconstructed by a process that engenders its own constraints (Montévil and Mossio, 2015). In short, we live as if we were running on a tight string, by continual adjusting the constraints on a critical trajectory; and organisms do it quite well, and so do species, at least for some time. There is no such thing as "equilibrium" in phylogenesis nor in ontogenesis: a non-artificial ecosystem is never in equilibrium, it is always evolving. Only death in a desert of stones is biological "equilibrium".

Many physicists work at co-evolutive dynamical systems in Biology and, by powerful mathematical tools, they try to model features like the ones above. The problem is that there is no predesigned space of phases, ${ }^{16}$ that is a space of the possible

\footnotetext{
14 As mentioned above, a tissue, an organ is not made by soft, inert matter, as it happens also when the latter is produced by an organism (e.g. wax cells or shells). Physical forces constrain the growth of a tissue, an organ, but they do not complete determine its shape, as in most existing models of morphogenesis, since Turing's 1952 seminal paper and Thom's work. Assuming first the Darwinian principle of "proliferation with variation" for cells, even within an organism, leads, in morphogenesis, to an original and more effective perspective also in modeling, (see Montévil et al., 2016b; Montévil, 2017).

15 This section as well as other parts authored by Longo in this paper, in particular the idea of "changing phase space" as for biological dynamics (see below), is taken from G. Longo "Space and Time in the Foundations of Mathematics, or some challenges in the interactions with other sciences". Invited lecture, First AMS/SMF meeting, Lyon, July 2001 (unpublished manuscript, downloadable; partly published in French, in Intellectica, 2003/1-2, n. 36-37).

16 The space of pertinent observables and parameters. This is the idea hinted in the 2001 paper quoted in the previous note.
}

phenotypes, where one could draw evolutionary geodesics: the phase space is co-constituted at the same time as the phenomenon to be described. Since Newton, as analyzed by Kant, one must give first the very conditions of possibility for the intelligibility of physical dynamics: the "a priori" of space and time. In the XIX century, these a priori for the physico-mathematical constructions where generalized to the notion of "phase space" (Hamilton, Gibbs, Poincaré ...), first given by position and momentum. Then the preliminary, difficult and fundamental task of the theoretician in physics became the invention of the suitable "phase space" for the intended analysis. This mathematical space moved from the position/momentum (or energy/time) space of classical dynamics to the more complex phase spaces of thermodynamics (Bailly and Longo, 2011). Note that the position/momentum and energy/time spaces of classical dynamics yield also the "conjugated variables" of quantum physics, where the indetermination of measurement was proposed. Later, an even more abstract complex Hilbert space, of infinite dimensions and where the quantum dynamics is the dynamics of an amplitude (a "law") of probability in Schrödinger's equation, (Bitbol, 1996).

The historicity of biological evolution is grounded on the changes of the very "phase space", that is on the changes of the pertinent Darwinian observables (phenotypes, thus organisms and species) and of the pertinent parameters, while rare events scan any phylogenetic trajectory, (Longo, 2017). These observables depend on each other, while interacting with billions of other phenomena, as unpredictable as the one above (the "latent potentials"). And this, along an extended critical state. Are there just "hidden variables", or missing parameters, to be discovered and inserted in the model? There seems to be more than this. Minor variations in the evolutive context, a mutation say, may create a new phase space: attractors which should describe the dynamics, not only need to be embedded in larger spaces (more variables), but seem to "swing" into different and co-constituted phase spaces. How to handle mathematically these changes, which may be "conceptual" changes? We are in a situation similar to the multiscale nature of biological phenomena, mentioned in $\S .7$ (and surely related to it), but with its own mathematical difficulties. Mathematics and its applications grew by proposing novel conceptual frames, as pointed out also throughout recent history by Patras (2001), possibly grounded in new forms of "access" to phenomena (in our sense above) or to new objects of knowledge.

We should try to give a rigorous interpretation of the gap between (the use of mathematics in) Physics and Biology, at least comparable to the one Quantum physicists proposed with regard to more classical approaches. Mathematics is an open conceptual construction and may be indefinitely enriched: fortunately, it is not God given, nor it is all already contained in and mechanically derivable from today's Zermelo-Fraenkel Set Theory or (predicative) fragments of Second Order Arithmetic, as some logicians still claim. When Newton and Leibniz unified metaphysically distinct universes, the sub-lunar and the supra-lunar bodies and their movements, they did not use the mathematics of projectiles well developed by the engineers of the time, largely based on Greek Geometry. They invented radically new concepts and tools, not contained in Euclid's notions and axioms, and dared to use the actual infinite to analyze finite movement (trajectories, speed, acceleration), a true revolution. Of course, there was a path through History, which led to their ideas, as we hinted, but the dynamics of mathematics swung by their work into a different conceptual space, which included infinitesimal analysis. And, by Gauss' and Riemann's Differential Geometry, this also changed Geometry.

In the XX century, from Dirac's delta function to Feymann's integral and Weyl's Gauge Theory or non-Commutative Geometry, radical mathematical novelty marked the construction of 
(Quantum) Physics. The difference is in knowledge construction and in the role of mathematics in it. We tried to point out some of the limits one encounters when treating biological entities with tools from Physics (see Longo, 2017 for more), similarly as the objects or the Geometry of Classical or Relativistic Physics do not apply to microphysics: either the objects become "strings", like in String Theory, or we need new understanding of space, like in nonCommutative Geometry. Also in Biology, we need new conceptual tools and possibly invent more suitable mathematics, as mathematicians and physicists did in Physics, from Newton to Connes. Typically, by their peculiar autonomy and contextual dependence, we cannot easily draw their mathematics on the phenomenal veil by "cutting them off" from their contexts and by giving them a constructed contour. The organismal unity seem to impose itself and the ecosystemic interactions produce the historical dynamics of the phase space. This, we believe, is the underlying methodological challenge for mathematics in Biology.

\section{Mathematical and epistemological projects}

In the spirit of this special issue, we will now hint to some possible work directions coming out from the proposed perspective. The central theme, on one side, aims at (re-)embedding mathematics and its constructive practices in phenomenal space and time, which mathematics contributes to constitute, also by the rich blend of the geometric perspective and the algebraic approach, since the encounter of the Greek and the Persian/Arabic traditions. On the other, space and time may relate to the very foundation of mathematics, which has been for too long isolated within the enclosed terms of its internal foundation, since Hilbert's Metamathematics is a mathematical discipline. By an enriched analysis of biological space and time, mathematics may better relate to this form of knowledge whose phenomenalities are first of all a spatiotemporal matter and require a synthetic insight on organisms.

\subsection{Role of Algebra and modern unifying perspectives}

In section one, we focused on the developments of Geometry from Euclid to Descartes, emphasizing the role of Algebra in this regard. We now draw on some comparisons between this tradition in Algebra beginning with al-Khwarizmi and the disciplines of Arithmetic and Geometry inherited from Babylonian and Greek traditions. The purpose is to stress the historical, methodological and conceptual, constitution of mathematics: when embedded in different historical contexts or when facing new challenges, mathematicians invented new tools and new mathematical structures, for the purposes of knowledge constructions. One of today's challenges is the analysis of biological processes, since Darwin's revolution, so far very poorly dealt with mathematical tools, or at most dealt with one century old tools from mathematical physics, even today, (see Longo, 2017). We need instead a similar creativity as the one hinted above in the very rich historical relation between mathematics and Physics or when dealing with practical needs. We review it here by some more hints towards a cross-cultural analysis.

The innovative role of Algebra in bridging the gap between two mathematical traditions of arithmetic and geometry provided a synthesis of mathematical entities that were considered to be otherwise independent: numbers and magnitudes. This synthesis was not the result of a mere conjunction of two inherited disciplines, rather a consequence of inventing a new mathematical entity altogether. This new entity, "the unknown", was so general that it allowed at once geometric and arithmetic determinations.

The invention of this new entity was a consequence of, in part, an ontologically neutral view of mathematics, which did not limit mathematics to pure, ideal objects of the Platonic realm nor an
Aristotelian knowledge construction. It was a completely different outlook on science or knowledge, which did not value the theoretical above the practical disciplines ("science" above "art", see the footnotes in sect. 1.3). On the contrary knowledge without action and action without knowledge were both strongly condemned. This "formal" ontology did not lower the status of Algebra to a mere technique, a collection of algorithms for finding solutions to equations but placed it between art and science. The algorithms were first (in work of al-Khwarizmi) carefully coupled with geometric demonstrations, and later with independent (algebraic) arguments for their soundness.

In doing Algebraic manipulations with the "unknown", one is invited to forget the properties of the entity to which the "unknown" refers and instead to focus on the relationships between different terms of the equation (known and unknown). In this way Algebraic manipulations are done at a more "abstract", "meta" level and the subject of mathematical study shifts from magnitudes to relations between them (and later to "structures" such as groups, rings, fields, with the rise of Abstract Algebra).

The effectiveness of this "formal" algebraic entity, "the unknown", first in legal matters and inheritance laws, as noted above, opened the way to further applications of mathematics in other sciences such as astronomy, mechanics, optics, etc. Gradually, with the scientific revolution of the 17th century it enriched the ontological commitment concerning the intrinsic mathematical nature of the Universe (as in Galileo) by the use of abstract algebraic computations. Algebra provided the formal tool for the expression of physical laws as relations between quantities of different kinds, replacing the geometric theory of proportions. The writing of the laws was based on observations and meaningful insights, and once laws were written the computations were done in formal algebraic or analytic fashion.

This ontologically neutral attitude led, among others, to the acceptance of zero and gradually to the development of an arithmetic for negative quantities. ${ }^{17}$ While conceiving zero encountered a fundamental obstacle with Greek ontological commitment as nature rejects the void, for algebraists it created no problem and was freely used by al-Khwarizmi in the system of Arabic numerals (base 10 ) that we still use today. ${ }^{18}$ As for negative numbers the process was slower partially because the algebraists didn't have a use for them. With the arithmetization of Algebra and the development of arithmetic for known and unknown quantities alike, as we discussed in 1.3 , negatives gradually came to be treated similar to positives (under arithmetical operations), also following the Brahmagupta tradition. ${ }^{19}$ The physician al-Samaw'al in the 12 th century completed the project that al-Karaji has started in the arithmetizaion of algebra: he defined algebraic power (including an unknown to the power zero) and recognized negatives as "deficiency" and positives as "excess" (Berggern, 2016). ${ }^{20}$

\footnotetext{
17 An early mention of negative numbers may be found in China, in the "Nine chapters on the art of mathematics" (Han Kingdom, 206 BCE - 220 AC): red strokes for positive numbers, black ones for negative. A more consistent use is made by the Indian mathematician Brahmagupta (598-668) in the Brahma Sphuta Siddhanta (628), where both negative numbers and 0 are posited jointly to their basic algebraic properties (Séguit-Duclot, 2017).

18 The first systematic treatment of Arabic numeral 1-9 and 0 and place-value system is in al-Khwarizmi's book "Hindu art of reckoning". While Hindus used the word "Sunya" (empty or blank), he introduced "sifr", "cipher".

19 Note that for the geometric algebraists such as Khayyam, who remained faithful to the necessity of geometric interpretation, accepting negatives was much more difficult.

${ }^{20}$ Al-Samw'al writes, ... if we subtract a deficient number from a deficient number larger than it, there remains the difference [e.g. $-5-(-2)=-(5-2)$ ], deficient; but in the other case there remains their difference, excess. [e.g. $-2-(-$ 5) $=+(5-2)] . "$ (Berggern, 2016).
} 
Moreover, the autonomy from the methods of Euclidean geometry, based on constructions by ruler and compass, led to the possibility of proving negative results: the "impossible" solutions. The Persian mathematician Nasir al-Din al-Tusi in the 12th century, for instance, in his book "The Equations", classified equations into two groups of possible and impossible (ones with no positive solution), and for the latter provided reasons as why these equations are "impossible cases". ${ }^{21}$ Of course, the "impossibility" of these equations is only the result of the limitation of the "world of Algebra" to real numbers (as we classify them today). With the invention of imaginary numbers (Cardano, XVI century) and of the geometric interpretation of complex numbers as vectors and points on the plane (Wessel, Wallis, Argand and Gauss, XVIII and XIX century), every polynomial turns out to be a "possible" equation. These representations made it possible to legitimize the use of these solutions, and to finally prove the fundamental theorem of Algebra for every polynomial of degree $n$ on the "complex plane" (Islami, 2017). It was in fact the dynamic interaction between algebraic and geometric methods that enriched mathematics and widened its possible applications.

While geometry, arithmetic and the theory of proportion were widely used in modern physics since its conception in the 17th century, Algebraic methods, jointly to analysis, gradually became the dominant (although not the only) language of physics in the 19 th century, all the while embedding physical processes in a sound geometry of space (the physicalization of geometry and the geometrization of physics we mentioned above). Typically, the requirement of homogeneity of magnitudes, after the introduction of (absolute) "unit", turned into the discipline of "dimensional" analysis, which is still of utter importance to the work of contemporary physicists. This is a complex blend of equational writing and a geometric appreciation of the notion of "dimension". Similarly, representations, by diagrams, graphs and trees etc, provided geometric insight and easier manipulations of combinatorial structures and algebraic operators; conversely, the general analysis of connected components and holes in surfaces was made possible by Betti numbers and Poincaré's groups, leading to homotopy and homology groups, as new algebraic tools for Geometry, (Dieudonné, 1972; Patras, 2014).

Let's finally recall the further unification by deep "transversal" concept and structures, more recently proposed by Grothendieck within pure mathematics, (see Zalamea, 2012 and Longo, 2015) for philosophical reflections (the second also in relation to Biology). This approach "goes across" Geometry, Algebra and Analysis, more than unifying them: the notion of Topos, say, as an extreme generalization of mathematical "space", applies transversally and sets the grounds for contemporary Algebraic Geometry.

\subsection{The epistemology of mathematics and the sciences of life}

There is no doubt that there are logic and pure formalisms, in mathematical proofs, yet their analyses are "necessary even though they are not sufficient" for a foundational project, as pointed out in Weyl (1927); that is, the belief in their mathematical, or even "cognitive", completeness is wrong, as we tried to argue. The analysis of the "mode of access" to phenomenal space, including the various forms of measurement, is an essential component of an epistemological reflection and contributes to open to new interactions between mathematics and empirical sciences. The $\mathrm{XX}$ century prevailing monomania of focusing only on the invariants of

\footnotetext{
21 Both Khayyam and al-Tusi come close to Descartes' and Fermat's results. Yet, without invention of a new method it was impossible for them to take these results further.
}

language and conscious reasoning (logic and formalisms), would be now a major limitation to further investigations. ${ }^{22}$

Concepts and structures are constituted in the interface between us and the world, on that phenomenal veil over which we draw them in order to organize and make intelligible the world, by mathematics. They originate on the regularities we "see", as living and historical beings, and develop along History, in intersubjectivity and language. These constructions are not arbitrary, as "reality" makes frictions and canalizes our knowledge dynamics. The common cognitive grounds, proper to our shared humanity, give generality and constitutive power to this procedure, enriched by historical specification and diversity. The objectivity of mathematics is in this process.

The reflections proposed above, concerning the challenges for mathematics in Biology, are not just meant as informal considerations, but they are an attempt to analyze the peculiar interface by which life presents itself to us. The mathematical analysis of the difficulties should stimulate a foundational investigation on the tools used and stress this constitutive role that mathematics has in knowledge construction: these difficulties are due to the different "autonomy", criticality and multiscalar phenomenality of life, if compared to the physical phenomena. In general, each analysis of the interface between us and phenomena, within different forms of knowledge or access to reality, bears a foundational character.

The focus on the issue of space is not meant to present a new monomania, that of Geometry, but to enrich existing paradigms by what was programmatically excluded by the founding fathers of modern Logic, and for good reasons (at their time: we are no longer troubled, today, by Riemann's Geometry and, perhaps, even not by Connes'). Moreover, the mathematics of space and time are "transversal" themes to different sciences; thus, the related foundational and methodological considerations should be an essential component of interdisciplinary researches. It is largely insufficient to transfer well-established techniques or algorithms from one discipline to another. We have to be "monist of matter" not of the "tools" for knowledge: different phenomenalities may need to be analyzed by different tools. Yet, an explicit reflection on the methodological differences and analogies may lead to a unification, which is never a matter of a transfer or superposition of techniques, but of a new invention, a new synthesis. Since Newton and Boltzmann mathematical physics proceeds by "unification", that is by the invention of a "third" theoretical frame unifying different phenomenalities - as for Newton, recall the example mentioned above of infinitesimal analysis and the role of infinity in it. Indeed, the same could be said for Boltzmann 's statistical physics that asymptotically correlated particles' trajectories and thermodynamical properties. But also Connes' non-commutative Geometry, a major advance forwards, unifies previous approaches, in between Relativistic and Quantum Physics, by new mathematical concepts. Grothendieck's "transversal" correlations, bypassing the frontiers of mathematical disciplines, is a further way to give unity to our major tools for scientific knowledge, mathematics, along the lines of the marriages we described in this paper.

The problem is opened on whether the immense conceptual creativity of mathematics may help to move from the "a priori" still

\footnotetext{
22 Even within formal systems, normalization for typed lambda-calculi (see Girard et al., 1989), as well as recent concrete incompleteness results, provide examples of very interesting formally unprovable theorems of Arithmetic: they use wellordering properties of numbers, as judgments of a "geometric" nature, in the proofs (see Longo, 2011a). Moreover, continuous "geometric" structures may step in the inductive load of proofs of purely combinatorial properties of computable functions and functionals (see "Four letters by Georg Kreisel" on this and subsequent work quoted in introduction to these letters, in http://www.di.ens.fr/users/ longo/files/FourLettersKreisel.pdf). Pure formalisms do not go very far.
} 
governing the relation between mathematics and physics (the pregiven phase space in each physico-mathematical theory) and invent the new mathematical concepts and structures needed for biology, possibly including a dynamics of the very phase space. The historical co-constitutive relation between mathematics and physics as well as the construction of mathematics for practical aims with no ontological commitments, have been very rich of these sorts of invention, as we hinted above. The conceptual frame for biology hinted in Longo and Montévil (2014), Soto and Longo (2016) and Longo (2017) is more radical as it is based on the proposal of new "a priori" in biological theorizing, inspired by observations and grounded on principles of organization and of variation, and on the peculiar, constitutive, role of historical time and rhythms for organisms. Scientific observations, of course, are not the contemplation of "empirical truth" nor "empirically real" (whatever this may mean), as they include the observer's choice of what and how to observe and measure, where to set the contours and how to qualify the objects of analysis. Then the challenge we tried to face is to "elevate" some crude observations (organisms, including cells within an organism, proliferate with variation and generate rhythms, along an historical time) to the status of founding conventions, as Poincaré would say, - or, to the status of "coordinating or constitutive principles", as soundly put in Friedman (2001), by revisiting Poincaré. If we go beyond the physicalist prejudices in Biology, an even richer path may be opened for new mathematics, with a similarly rich constitutive power than the one physics has had for mathematics.

Theorizing or even philosophical reflections (both ontological and epistemological) need to precede the mathematization, if the latter is ever possible, as it was the case in classical and relativistic physics, since Galileo and Descartes or Riemann and Mach, or in quantum mechanics, where the theoretical debate always had and has deep philosophical implications and preceded mathematization. This is the attempt, as for a theory of biological ontogenesis which precedes mathematical modeling. Some original modeling techniques are already being designed following that theoretical approach, as for organ morphogenesis, (Montévil et al., 2016b; Montévil, 2017), typically by considering conceptually primary the proliferation with variation of cells then the tissular dynamics under the constraints of physical forces. Moreover, new, non obvious mathematics is being proposed, partly inspired by the philosophical perspective mentioned here in biology, concerning the changes of the very phase space, (see Sarti and Citti, 2017).

\section{Acknowledgements}

Islami would like to thank Thomas Ryckman, Michael Friedman, Tom Donaldson, Ladislav Kvasz and Harald Wilstche for immensely helpful discussions. Longo's activity in two working groups (CeSEF, on the epistemology of Quantum Physics) and 'Géométrie et Cognition', has been a fantastic occasion to collaborate with several colleagues in Physics, Philosophy and Biology, in Paris. Longo is particularly indebted to the joint work and uncountably many discussions with his students Maël Montévil, Matteo Mossio and Nicole Perret, as well as with Francis Bailly, Jean Petitot, Bernard Teissier, Mioara Mugur-Schachter, Ana Soto, Carlos Sonnenchein.

\section{References}

Al-Khwarizmi, A.J. 1915, (813?) Kitab al-Jabr va al-Moquabala.

Bailly, F., Longo, G., 2011. Mathematics and the Natural Sciences: the Physical Singularity of Life. Imperial College Press, London (original French version, Hermann, 2006).

Bailly, F., Gaill, F., Mosseri, R., 1994. "Morphogenèse et croissance biologique : un modele dynamique simple pour le poumon" dans La biologie théorique a Solignac. Ed. Polytech. 65-94.
Berggern, J.L., 2016. Episodes in the Mathematics of Medieval Islam. Springer-Verlag New York.

Bitbol, M., 1996. Schrödinger's Philosophy of Quantum Mechanics. Kluwer, Dordrecht; Boston.

Bizzarri, M., Cucina, A., Palombo, A., Masiello, M.G., 2014. Gravity sensing cells: mechanisms and theoretical grounds. Rend. Fis. Acc. Lincei 25, S29-S38.

Boi, L., 1995. Le problème mathématique de l'espace. Springer.

Bottazzini U., Poincaré, Le Scienze, 1999.

Bottazzini, U., Tazzioli, R., 1995. Naturphilosophie and its role in Riemann's mathematics. Rev. d'Histoire Des. Mathématiques n 1, 3-38.

Brown, J.H., West, G., 1999. Scaling in Biology. Santa Fe Institute Publication.

Châtelet, G., 1993. Les Enjeux du Mobile. Seuil, Paris.

Connes, A., 1994. Non-commutative Geometry. Academic Press.

Descartes, R., 1954. La Géométrie 1637, English Translation: the Geometry of Rene Descartes. Dover, New York.

Dieudonné, J., 1972. The historical development of algebraic geometry. Am. Math. Mon. 79 (8), 827-866.

Ehresmann, A.C., Vanbremeersch, J.-P., 2007. Memory Evolutive Systems: Hierarchy, Emergence, Cognition. Elsevier Science.

Frege, G. 1873. On a geometrical representation of imaginary forms in the plane. In: McGuiness, B. (Ed.), Collecteed Papers on Mathematics, Logic and Philosophy. Basil Blackwell, Oxford (1984).

Frege, G., 1884. The Foundations of Arithmetic (english transl. Evanston, 1980).

Friedman, M., 2001. Dynamics of Reason. Stanford CSLI publications.

Girard, J.Y., Lafont, Y., Taylor, R., 1989. Proofs and Types. Cambridge University Press. Gould, S.J., 1982. The Panda's Thumb. WW. Norton.

Gould, S.J., 1989. Wonderful Life. WW. Norton.

Heath, T.L., 1908. The Thirteen Books of Euclid's Elements. Cambridge Univ. Press. Heinzmann, G., 1998. Poincaré on Understanding Mathematics. Philosophia Scientiae, pp. $143-160$.

Hilbert, 1899. Les fondements de la géométrie (trad. fran., Dunod, 1971).

Husserl, E., 1933. The Origin of Geometry.

Husserl, E., 1936. The Crisis of European Sciences and Transcendental Phenomenology.

Islami, A., 2017. "A Complex Problem for Formalists", to appear.

Jennings, C., Aamodt, S., November 2000. Computational approaches to brain functions. Nat. Neurosci. Suppl. 3.

Kvasz, L., 2008. Patterns of Change: Linguistic Innovations in the Development of Classical Mathematics. Birkhäuser Verlag.

Leibniz, G.W., 1686. Translated by. In: Lucas, P.G., Grint, L. (Eds.), Discourse on Metaphysics. Manchester University Press, Manchester, 1953.

Longo, G., 2011a. Reflections on concrete incompleteness. Philos. Math. 19 (3), $255-280$.

Longo, G., 2011b. Theorems as constructive visions. In: Hanna, de Villiers (Eds.) Invited Lecture, Proceedings of ICMI 19 Conference on Proof and Proving. Springer, Taipei, Taiwan. May 10-15, 2009.

Longo, G., December 2015. Conceptual Analyses from a Grothendieckian Perspective: Reflections on Synthetic Philosophy of Contemporary Mathematics by Fernando Zalamea in Speculations. https://www.urbanomic.com/speculations_ lo/.

Longo, G., 2017. How future depends on past histories and rare events in systems of life. Found. Sci. https://doi.org/10.1007/s10699-017-9535-x.

Longo, G., Montévil, M., 2014. Perspectives on Organisms: Biological Time, Symmetries and Singularities. Springer, Dordrecht.

Longo, G., Montévil, M., Pocheville, A., 2012. From bottom-up approaches to levels of organization and extended critical transitions. Front. Physiol. 3, 232.

Montévil, M., 2017. A primer on mathematical modeling in the study of organisms and their parts. In: Bizzarri (Ed.), Conceptual and Methodological challenges in Systems Biology. Springer. Methods in Molecular Biology.

Montévil, M., Mossio, M., 2015. Biological organisation as closure of constraints. J. Theor. Biol. 372, 179-191.

Montévil, M., Mossio, M., Pocheville, A., Longo, G., 2016a. Theoretical principles for biology: Variation. Prog. Biophys. Mol. Biol. 122, 36-50. Soto, Longo.

Montévil, M., Speroni, L., Sonnenschein, C., Soto, A.M., 2016b. Modeling mammary organogenesis from biological first principles: cells and their physical constraints. Prog. Biophys. Mol. Biol. 122, 58-69. Soto, Longo.

Nabonnand, P., 2000. Les recherches sur l'oeuvre de Poincaré. Gaz. Des. Mathématiciens 85. Juillet.

Nabonnand, P., 2001. "La génèse psycho-physiologique de la géométrie selon Poincaré", à paraître dans La revue d'Histoire des Mathématiques.

Nonnenmacher, T.F., Losa, G.A., Weibel, E.R., 1994. Fractals in Biology and Medicine. Birkhauser.

Panza, M., 2011. Rethinking geometrical exactness. Hist. Math. 38, 42-95.

Panza, M., 2012. The twofold role of diagrams in Euclid's plane geometry. Synthese 186, 55-102.

Panza, M., 2015. “"'Una stessa cosa”. Come intendere la definizione della continuità di Aristotele", Fisica V.3, 227a10-12. In: Generali, D. (Ed.), Le Radici della razionalit'a Critica : saperi, pratiche, teleologie. Studi offerti a Fabio Minazzi, Mimesis, pp. 715-728.

Patras, F., 2001. La Pensée Mathématique Contemporaine. Press Univ. de France.

Patras, F., 2014. La possibilité des nombres. Presse Univ. France, Paris.

Petitot, J., Varela, F., Pachoud, B., Roy, J.-M., 1999. Naturalizing Phenomenology: issues in comtemporary Phenomenology and cognitive sciences. (J. Petitot al., eds) Stanf. U.P.

Poincaré, H., 1899. Des fondements de la géométrie. Revue de métaphysique morale 
7, 251-279.

Poincaré, H., 1902. La Science et l'Hypothèse. Flammarion, Paris.

Poincaré, H., 1913. Dernières Pensées. Flamarion, Paris.

Rahman, S., Street, T., Tahiri, H., 2008. The Unity of Science in the Arabic Tradition. Springer.

Rashed, R., 1996. Encyclopedia of the History of Arabic Science, vol. 2.

Rashed, R., 2015. Classical Mathematics from Al-Khwarizmi to Descartes. Routledge. Riemann, B., 1854. On the hypothesis which lie at the basis of Geometry (english transl. by W. Clifford, Nature, 1873.

Rosenthal, F., 1970. Knowledge Triumphant: the Concept of Knowledge in Medieval Islam. E.J. Brill, Leiden.

Sarti, A., Citti, G., 2017. Heterogenesis and the Constitution of Semiotic Spaces. Semiotica, submitted.

Séguit-Duclot, A., 2017. Défondation des Mathématiques, to appear.

Simeonov, P.L., Ehresmann, A.C., 2017. Some Resonances between Eastern Thought and Integral Biomathics in the Framework of WLIMES. this volume.

From the century of the genome to the century of the organism: new theoretica approaches. Special issue. In: Soto, A., Longo, G. (Eds.), Prog. Biophys. Mol. Biol. 122.

Tappenden, J., March 1995. Geometry and generality in Frege's philosophy of Arithmetic. Synth. n. 3102.

Turing, A., 1950. Computing Machines and Intelligence. Mind, LIX.

Turing, A., 1952. The chemical basis of morphogenesis. Philo. Trans. R. Soc. B237 $37-72$.

Vernant, J.-P., 1983. Myth and Thought among the Greeks. M.I.T. Press, Cambridge, USA.

Weyl, H., 1927. Philosophy of Mathematics and of Natural Sciences. English transl. Princeton University Press, Princeton, New Jersey, 1949.

Zalamea, F., 2012. Synthetic Philosophy of Contemporary Mathematics. Urbanomic and Sequence Press, NY. 